2 Figure DR1A. Displacement profiles for individual faults in the Niger Delta (NDN). All plots are

3 five times vertically exaggerated. For NDN2, lower, middle, and upper horizon levels were

4 mapped. Gray boxes at the tips represent uncertainties in the exact positions of lateral fault

5 terminations. The shapes of displacement profiles are extrapolated into these regions.

6 Figure DR1B. Displacement profiles for individual faults in the Sichuan Basin (SBS and SBN).

7 All plots are five times vertically exaggerated. For SBN6, SBS2, and SBS4, both lower and

8 upper horizon levels were mapped. Gray boxes at the tips represent uncertainties in the exact

9 positions of lateral fault terminations. The shapes of displacement profiles are extrapolated into

10 these regions.

11 Figure DR1C. Displacement profiles for individual faults in the Magdalena Basin (MBS and $12 \mathrm{MBN}$ ). All plots are five times vertically exaggerated. Gray boxes at the tips represent

13 uncertainties in the exact positions of lateral fault terminations. The shapes of displacement

14 profiles are extrapolated into these regions.

15 Table DR1. Displacement-length scaling $\left(D_{\max } v . L\right)$ data. $D_{\max }$ interpreted is the maximum 16 displacement from our preferred fault interpretation. Maximum error is the highest permissible

17 displacement from the seismic reflection data. Minimum error is the lowest permissible

18 displacement from the seismic reflection data. 

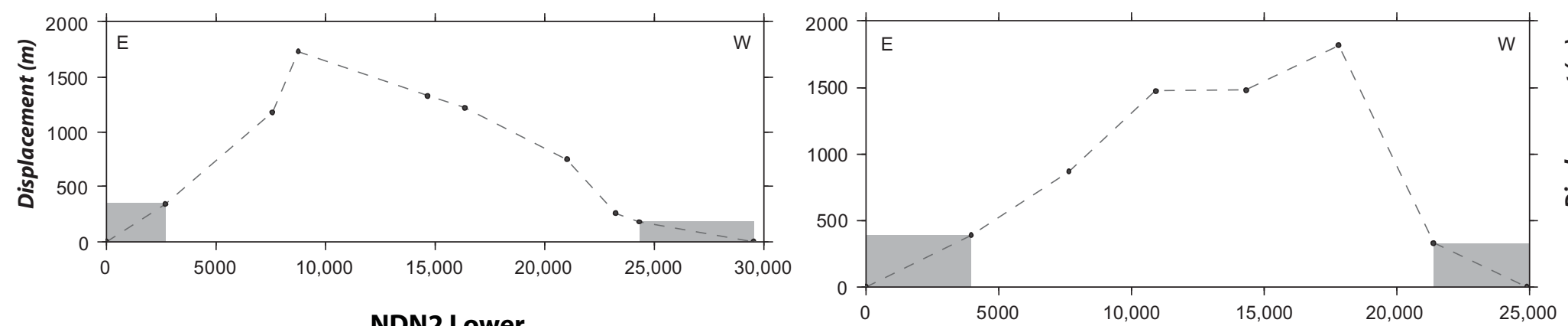

NDN2 Lower
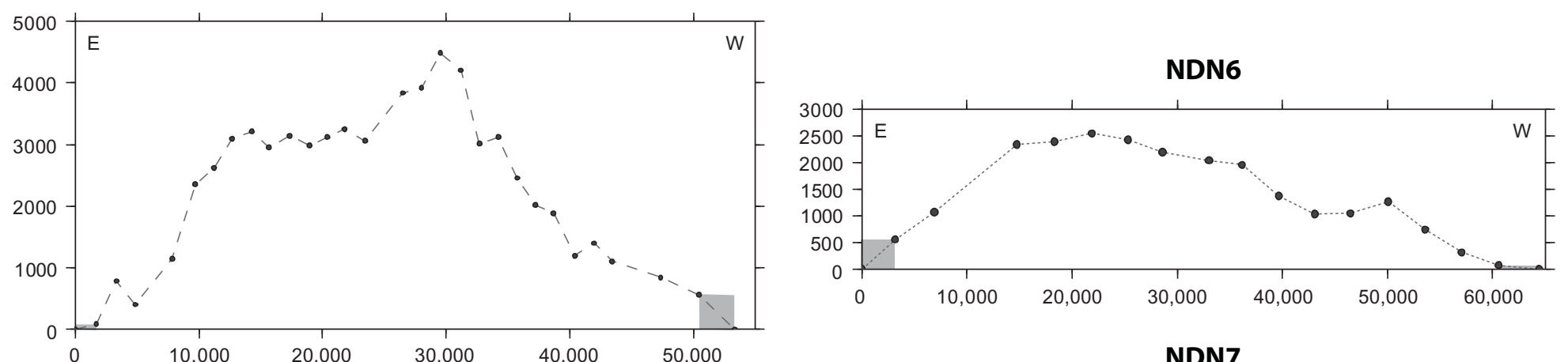

NDN7
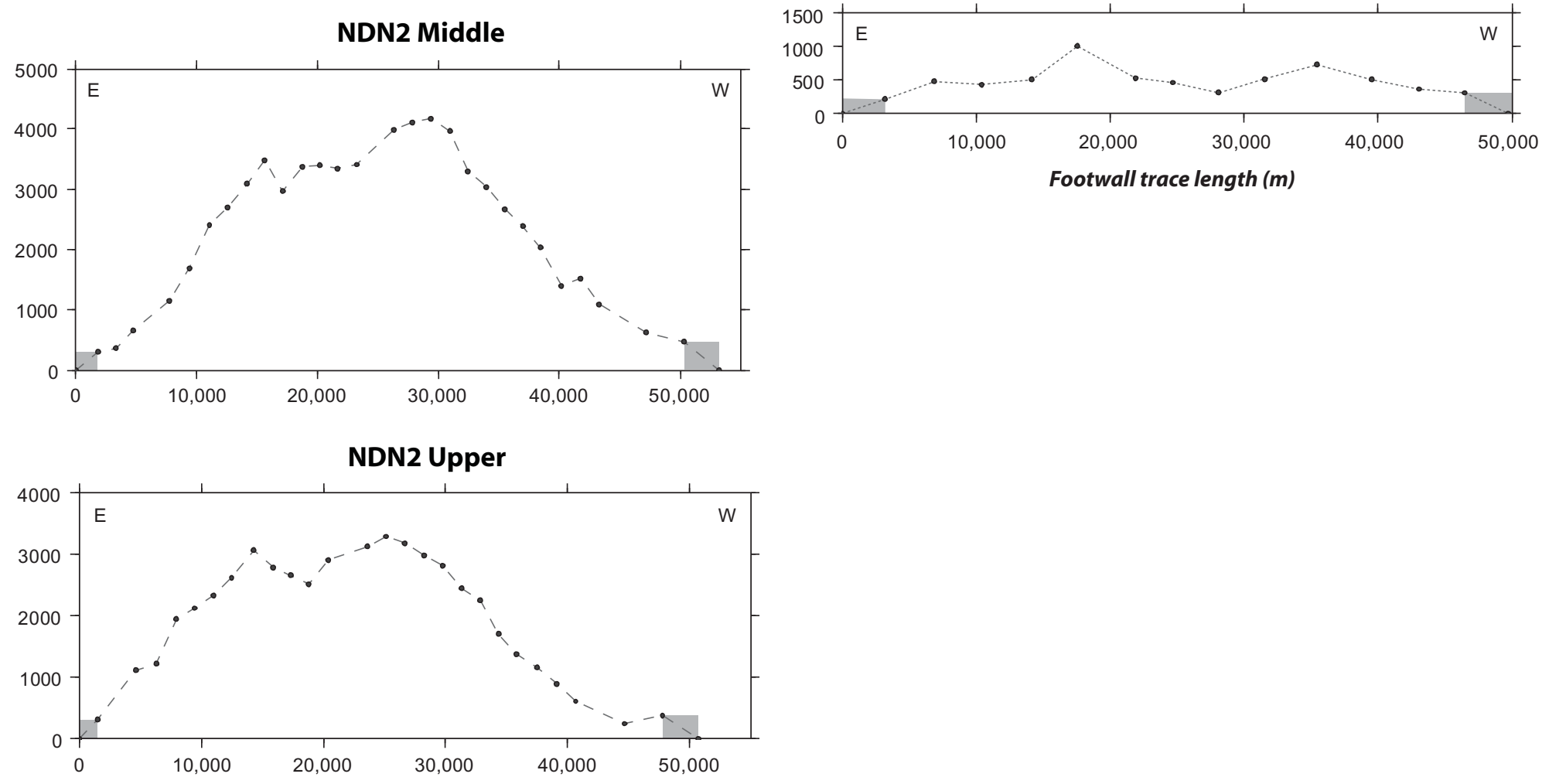

NDN3

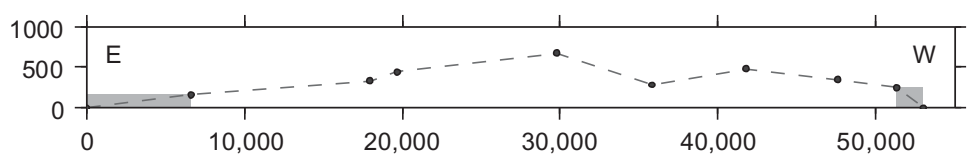

NDN4

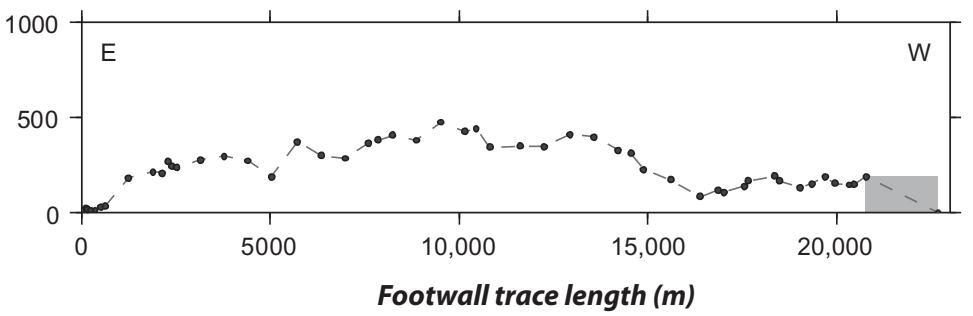



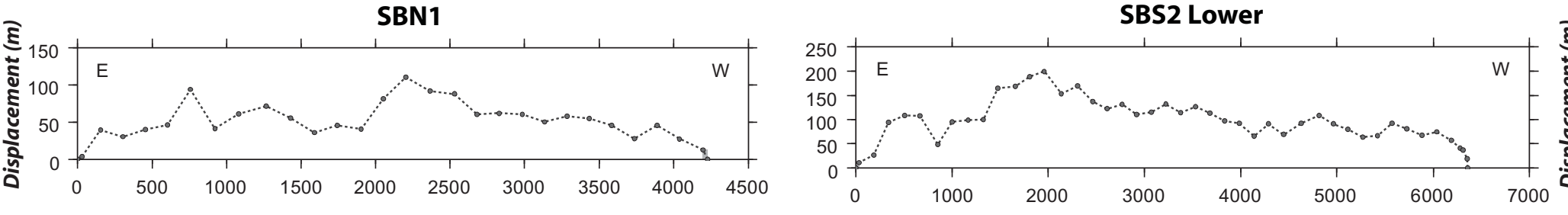

SBN2

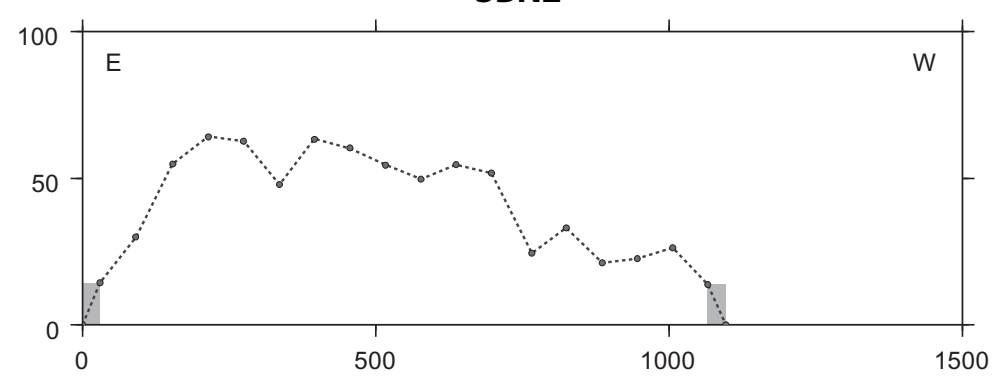

SBN3

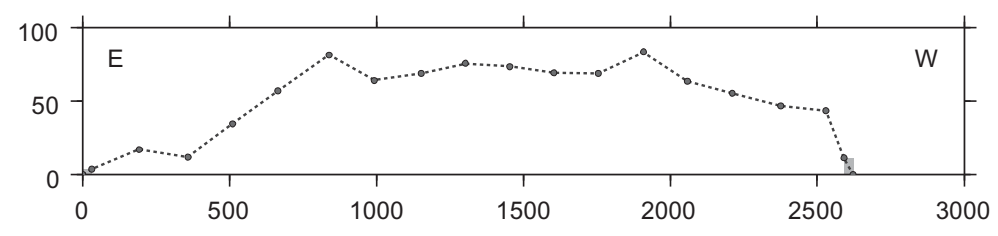

SBN4
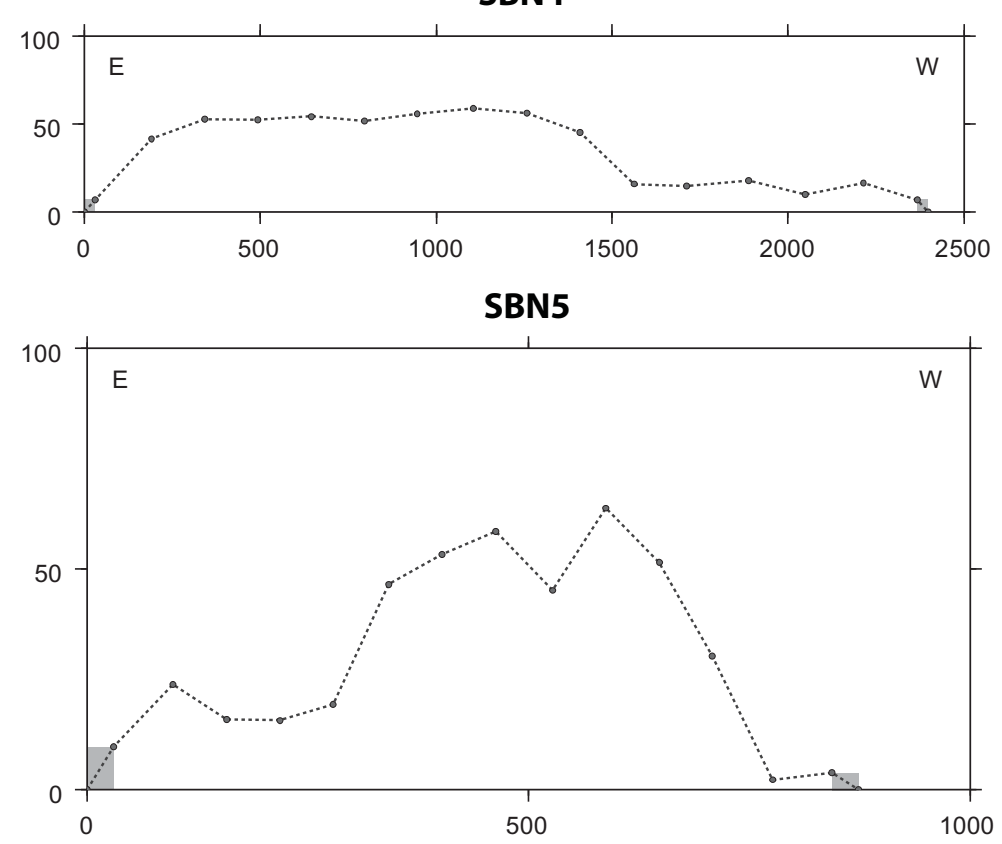

SBN6 Lower

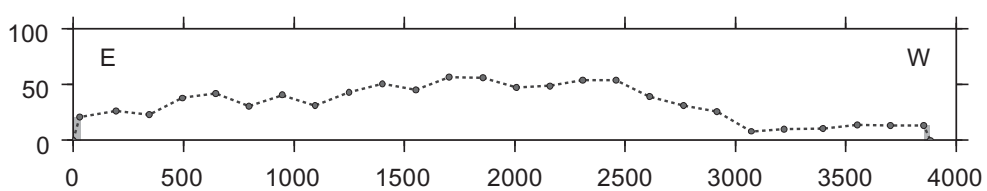

SBN6 Upper

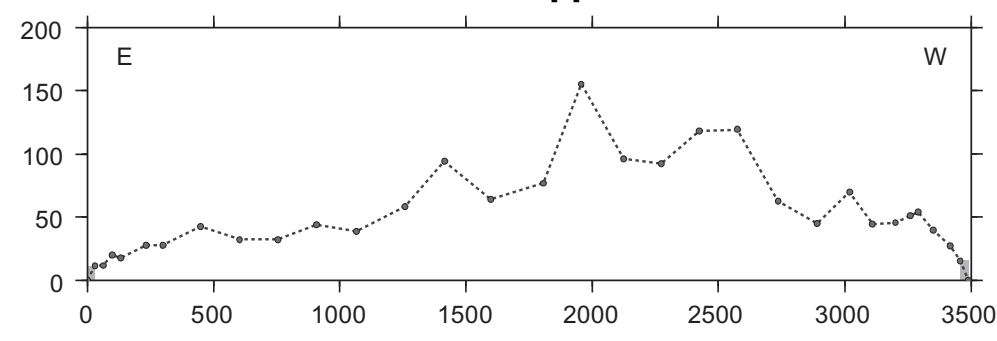

Footwall trace length $(m)$
SBS2 Upper

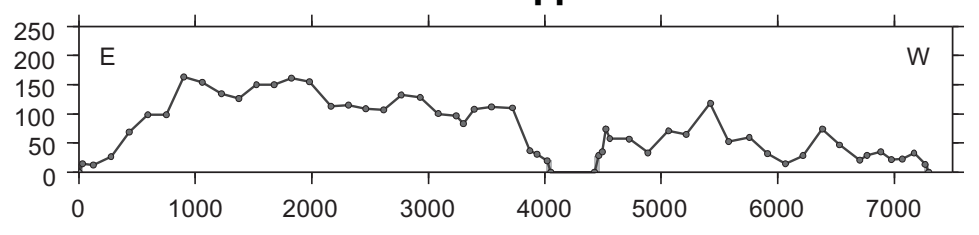

SBS3

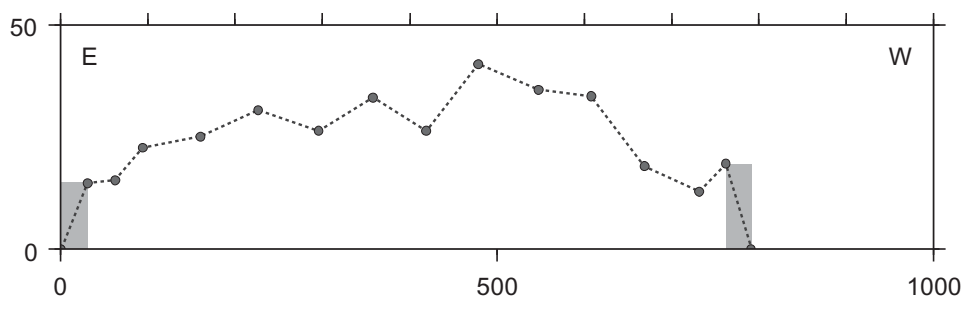

SBS4 Lower

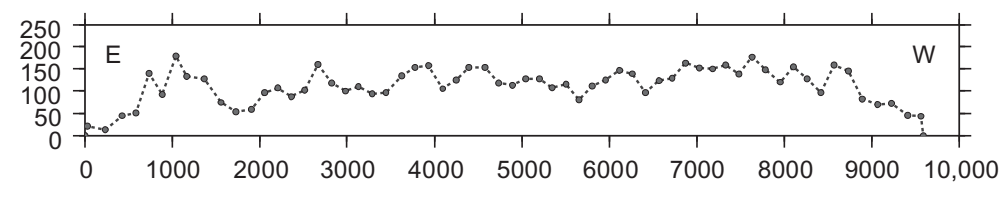

\section{SBS4 Upper}

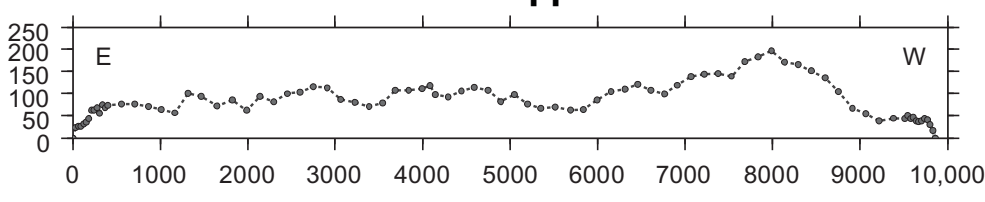

SBS5

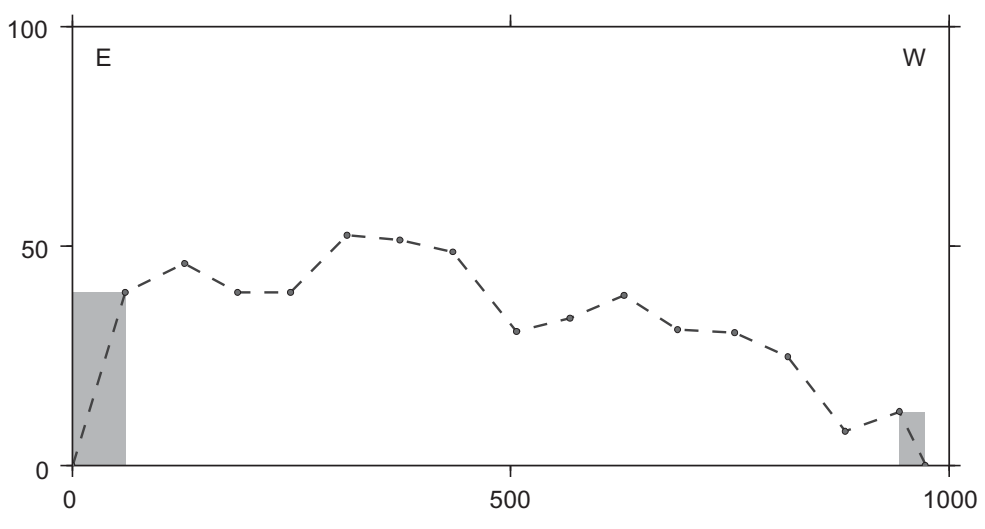

SBS6

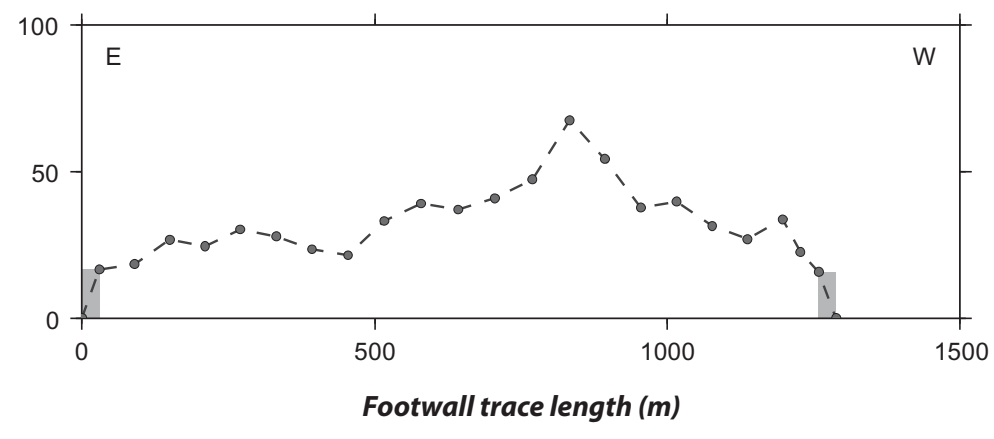

Bergen and Shaw, Figure DR $1 b, p d f$ 
MBN1

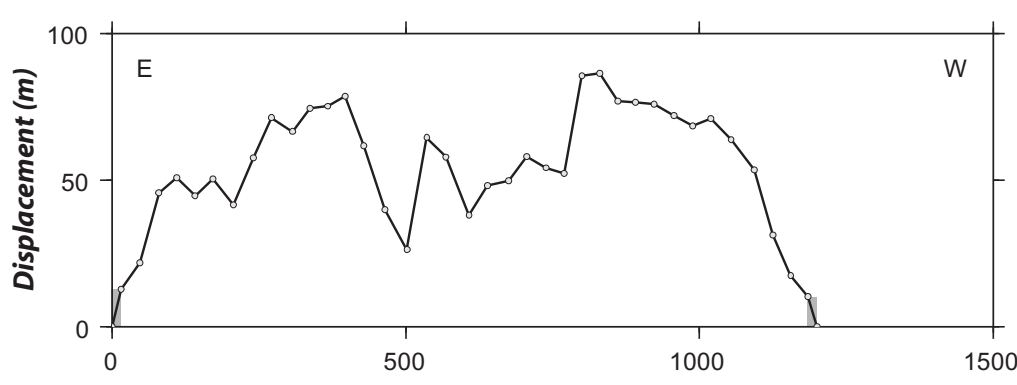

MBN2
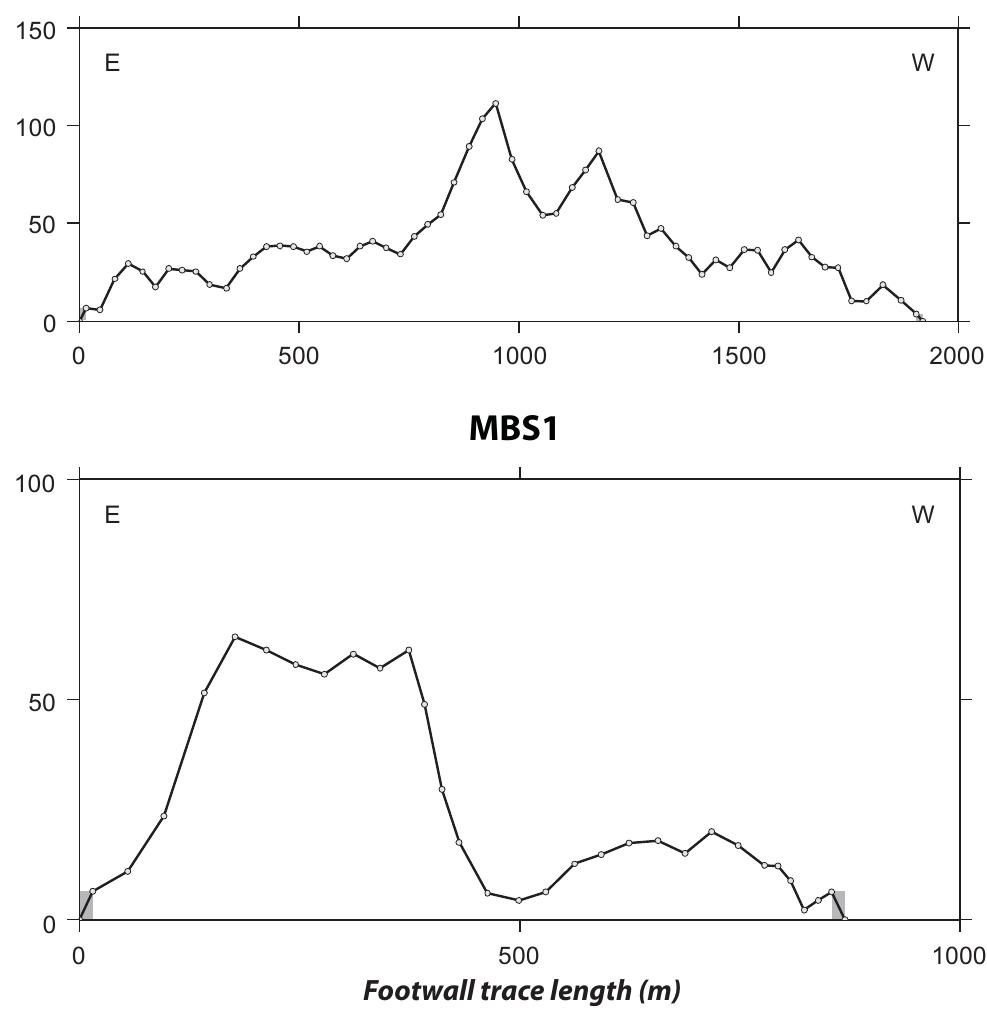

Bergen and Shaw, Figure DR1c, pdf 
Table DR1. $D_{\max }-L$ data

Fault Length $(m) \quad D_{\max }$ interpreted $(m) \quad$ Maximum Error $(m) \quad$ Minimum Error $(m)$

$\begin{array}{lcccc}\text { MBN1 } & 869 & 64 & 30 & 9 \\ \text { MBN2 } & 1919 & 112 & 46 & 25 \\ \text { MBS1 } & 1201 & 86 & 40 & 30 \\ \text { NDN1 } & 29537 & 1731 & 85 & 277 \\ \text { NDN2 } & 53341 & 4487 & 285 & 293 \\ \text { NDN3 } & 53025 & 677 & 94 & 68 \\ \text { NDN4 } & 22691 & 474 & 21 & 100 \\ \text { NDN5 } & 24905 & 1822 & 183 & 218 \\ \text { NDN6 } & 64376 & 2627 & 58 & 242 \\ \text { NDN7 } & 49732 & 1003 & 176 & 72 \\ & & & & 2 \\ \text { SBN1 } & 4226 & 110 & 30 & 19 \\ \text { SBN2 } & 1096 & 64 & 1 & 21 \\ \text { SBN3 } & 2621 & 83 & 15 & 18 \\ \text { SBN4 } & 2398 & 59 & 6 & 16 \\ \text { SBN5 } & 874 & 64 & 6 & 56 \\ \text { SBN6 } & 3488 & 155 & 32 & 17 \\ \text { SBS2 } & 6356 & 199 & 19 & 1 \\ \text { SBS3 } & 791 & 37 & 25 & 13 \\ \text { SBS4 } & 9590 & 179 & 22 & 14 \\ \text { SBS5 } & 943 & 52 & 1 & 11 \\ \text { SBS6 } & 1289 & 68 & 39 & \end{array}$

Bergen and Shaw, Table DR1, pdf 